\title{
New Leading Contribution to Neutrinoless Double- $\beta$ Decay
}

\author{
Vincenzo Cirigliano, ${ }^{1}$ Wouter Dekens, ${ }^{1}$ Jordy de Vries, ${ }^{2}$ Michael L. Graesser, ${ }^{1}$ \\ Emanuele Mereghetti, ${ }^{1}$ Saori Pastore, ${ }^{1}$ and Ubirajara van Kolck ${ }^{3,4}$ \\ ${ }^{1}$ Theoretical Division, Los Alamos National Laboratory, Los Alamos, New Mexico 87545, USA \\ ${ }^{2}$ Nikhef, Theory Group, Science Park 105, 1098 XG Amsterdam, The Netherlands \\ ${ }^{3}$ Institut de Physique Nucléaire, CNRS/IN2P3, Université Paris-Sud, Université Paris-Saclay, 91406 Orsay, France \\ ${ }^{4}$ Department of Physics, University of Arizona, Tucson, Arizona 85721, USA
}

(Received 1 March 2018; revised manuscript received 28 March 2018; published 16 May 2018)

\begin{abstract}
Within the framework of chiral effective field theory, we discuss the leading contributions to the neutrinoless double-beta decay transition operator induced by light Majorana neutrinos. Based on renormalization arguments in both dimensional regularization with minimal subtraction and a coordinatespace cutoff scheme, we show the need to introduce a leading-order short-range operator, missing in all current calculations. We discuss strategies to determine the finite part of the short-range coupling by matching to lattice QCD or by relating it via chiral symmetry to isospin-breaking observables in the two-nucleon sector. Finally, we speculate on the impact of this new contribution on nuclear matrix elements of relevance to experiment.
\end{abstract}

DOI: 10.1103/PhysRevLett.120.202001

Introduction.-Neutrinoless double-beta decay $(0 \nu \beta \beta)$ is the most sensitive laboratory probe of lepton number violation (LNV). In $0 \nu \beta \beta, L$ is violated by two units when two neutrons in a nucleus turn into two protons, with the emission of two electrons and no neutrinos. The observation of $0 \nu \beta \beta$ would demonstrate that neutrinos are Majorana fermions [1], shed light on the mechanism of neutrino mass generation [2-4], and give insight into leptogenesis scenarios for the generation of the matterantimatter asymmetry in the Universe [5].

$0 \nu \beta \beta$ is actively being searched for in a number of eveneven nuclei for which single- $\beta$ decay is energetically forbidden. Current experimental limits [6-15] on the half-lives are at the level of $T_{1 / 2}>5.3 \times 10^{25} \mathrm{y}$ for ${ }^{76} \mathrm{Ge}$ [12] and $T_{1 / 2}>1.07 \times 10^{26}$ y for ${ }^{136} \mathrm{Xe}$ [10], with nextgeneration ton-scale experiments aiming at improvements in sensitivity by two orders of magnitude.

$0 \nu \beta \beta$ can be generated by a variety of dynamical LNV mechanisms, which, in an effective field theory (EFT) approach to new physics, are parametrized by $\Delta L=2$ operators of odd dimension greater than 4 [16-22]. If the mass scale associated with LNV is much higher than the electroweak scale, the only low-energy manifestation of this new physics is a Majorana mass for light neutrinos, encoded in a single gauge-invariant dimension-five

Published by the American Physical Society under the terms of the Creative Commons Attribution 4.0 International license. Further distribution of this work must maintain attribution to the author(s) and the published article's title, journal citation, and DOI. Funded by SCOAP. operator [16], which induces $0 \nu \beta \beta$ through light Majorana-neutrino exchange $[23,24]$. To interpret positive or null $0 \nu \beta \beta$ results in this minimal scenario, it is crucial to have good control over the relevant hadronic and nuclear matrix elements. Current knowledge of these is not satisfactory [25], as various many-body approaches lead to estimates that differ by a factor of $2-3$ and most calculations are not based on a modern EFT analysis. In Ref. [26], a first step was presented towards the analysis of $0 \nu \beta \beta$ induced by a light Majorana neutrino in the chiral EFT framework [27-29], which provides a systematic expansion of hadronic amplitudes in $p / \Lambda_{\chi}$, where $p \sim$ $m_{\pi} \sim k_{F} \sim \mathcal{O}(100 \mathrm{MeV})$ and $\Lambda_{\chi} \sim 4 \pi F_{\pi} \sim m_{N} \sim \mathcal{O}(1 \mathrm{GeV})$. The $0 \nu \beta \beta$ transition operators were derived up to nextto-next-to-leading order $\left(\mathrm{N}^{2} \mathrm{LO}\right)$ in Weinberg's powercounting scheme [30,31].

In this Letter, we demonstrate that Weinberg's scheme for $0 \nu \beta \beta$ assumed in Ref. [26] breaks down and any consistent power counting requires a leading-order (LO) short-range $\Delta L=2$ operator, whose effect is missing in all current calculations. Our argument is based on renormalization. Using two different schemes (dimensional regularization with minimal subtraction and a coordinate-space cutoff), we show that, once the strong nucleon-nucleon scattering amplitude is made finite and independent of the ultraviolet regulator, an additional $\Delta L=2$ contact operator with coupling $g_{\nu}^{\mathrm{NN}}$ has to be introduced to make the $n n \rightarrow$ ppee amplitude finite and regulator independent. The finite part of $g_{\nu}^{\mathrm{NN}}$, which encodes hard-neutrino exchange, can be determined by (i) matching the chiral EFT $n n \rightarrow$ ppee amplitude to future lattice QCD 
calculations and (ii) relating it via chiral symmetry to electromagnetic low-energy constants (LECs) that control isospin breaking in the two-nucleon sector. A combination of couplings involving $g_{\nu}^{\mathrm{NN}}$ can be fit to nucleon-nucleon charge-independence-breaking (CIB) observables, confirming the LO scaling of this coupling. Based on this, we argue that the impact of $g_{\nu}^{\mathrm{NN}}$ on nuclear matrix elements of relevance to experiments can be significant, which has consequences for the interpretation of $0 \nu \beta \beta$ experiments in terms of the light Majorana-neutrino mass.

The need for a $L O$ short-range $\Delta L=2$ interaction.-We consider a scenario in which LNV at low energy is dominated by the electron-neutrino Majorana mass

$$
\mathcal{L}_{\Delta L=2}=-\frac{m_{\beta \beta}}{2} \nu_{e L}^{T} C \nu_{e L},
$$

where $C=i \gamma_{2} \gamma_{0}$ denotes the charge conjugation matrix.

The nuclear effective Hamiltonian can be written as

$$
H_{\text {eff }}=H_{\text {strong }}+2 G_{F}^{2} V_{u d}^{2} m_{\beta \beta} \bar{e}_{L} C \bar{e}_{L}^{T} V_{\nu},
$$

in terms of the Fermi constant $G_{F}$ and the $V_{u d}$ element of the Cabibbo-Kobayashi-Maskawa matrix [32,33]. The neutrino potential $V_{\nu}$ can be obtained from two-nucleon irreducible diagrams mediating $n n \rightarrow$ ppee to a given order in $p / \Lambda_{\chi}$. Within Weinberg's power counting, the only LO contribution [26] comes from the exchange of potential neutrinos, with $q^{0} \ll|\mathbf{q}|$,

$$
\begin{aligned}
V_{\nu, 0}(\mathbf{q})= & \tau^{(1)+} \tau^{(2)+} \frac{1}{\mathbf{q}^{2}}\left(1-g_{A}^{2} \boldsymbol{\sigma}^{(1)} \cdot \boldsymbol{\sigma}^{(2)}\right. \\
& \left.+g_{A}^{2} \boldsymbol{\sigma}^{(1)} \cdot \mathbf{q} \boldsymbol{\sigma}^{(2)} \cdot \mathbf{q} \frac{2 m_{\pi}^{2}+\mathbf{q}^{2}}{\left(\mathbf{q}^{2}+m_{\pi}^{2}\right)^{2}}\right),
\end{aligned}
$$

where $g_{A} \simeq 1.27$ is the nucleon axial coupling, $m_{\pi}$ is the pion mass, and $\mathbf{q}$ is the momentum transfer. $\mathrm{N}^{2} \mathrm{LO}$ terms arise from corrections to the single nucleon weak currents, irreducible one-loop diagrams, and contact interactions mediating $\pi \pi \rightarrow e e, n \rightarrow p \pi^{+} e e$, and $n n \rightarrow$ ppee. In particular, the short-range potential includes a two-nucleon term [26]

$$
V_{\nu, C T}=-2 g_{\nu}^{\mathrm{NN}} \tau^{(1)+} \tau^{(2)+},
$$

where the $\operatorname{LEC} g_{\nu}^{\mathrm{NN}}$ is $\mathcal{O}\left(\left(4 \pi F_{\pi}\right)^{-2}\right)$ in Weinberg's counting and $F_{\pi}=92.2 \mathrm{MeV}$ is the pion decay constant. However, it is known that Weinberg's power counting leads to inconsistent results in nucleon-nucleon scattering [34-37] and nuclear processes mediated by external currents [38], due to a conflict between naive dimensional analysis and nonperturbative renormalization. We therefore investigate the scaling of $g_{\nu}^{\mathrm{NN}}$ by studying the amplitude $\mathcal{A}(n n \rightarrow$ ppee $) \equiv \mathcal{A}_{\Delta L=2}$ with strong interactions $H_{\text {strong }}$ included nonperturbatively.

We work at LO in chiral EFT and focus on the scattering of two neutrons to two protons in the ${ }^{1} S_{0}$ wave, where $H_{\text {strong }}$ has short-range and Yukawa components,
$V_{0}(\mathbf{q})=\tilde{C}+V_{\pi}(\mathbf{q}), \quad V_{\pi}(\mathbf{q})=-\frac{g_{A}^{2}}{4 F_{\pi}^{2}} \frac{m_{\pi}^{2}}{\mathbf{q}^{2}+m_{\pi}^{2}}$,

with $\tilde{C} \sim \mathcal{O}\left(F_{\pi}^{-2}, m_{\pi}^{2} F_{\pi}^{-4}\right)[31,34,35]$. We have checked that transitions involving higher partial waves such as ${ }^{3} P_{0,1} \rightarrow{ }^{3} P_{0,1}$ are correctly renormalized and do not require enhanced $\Delta L=2$ counterterms.

The contributions to $\mathcal{A}_{\Delta L=2}$ from the exchange of a light neutrino $\left(\mathcal{A}_{\Delta L=2}^{(\nu)}\right)$ are shown in Fig. 1. The blue ellipse denotes the iteration of the Yukawa potential $V_{\pi}(\mathbf{q})$. The diagrams in the second and third rows include an infinite number of bubbles, dressed with iterations of $V_{\pi}$. Without loss of generality for our arguments, we use the kinematics $n(\mathbf{p}) n(-\mathbf{p}) \rightarrow p\left(\mathbf{p}^{\prime}\right) p\left(-\mathbf{p}^{\prime}\right) e\left(\mathbf{p}_{e 1}=0\right) e\left(\mathbf{p}_{e 2}=0\right)$, with $|\mathbf{p}|=$ $1 \mathrm{MeV}$ and, correspondingly, $\left|\mathbf{p}^{\prime}\right|=38 \mathrm{MeV}$.

$\mathcal{A}_{\Delta L=2}^{(\nu)}$ can be expressed in terms of the Yukawa "in" and "out" wave functions $\chi_{\mathbf{p}}^{ \pm}(\mathbf{r})$ and the propagators $G_{E}^{ \pm}\left(\mathbf{r}, \mathbf{r}^{\prime}\right)=$ $\left\langle\mathbf{r}^{\prime}\left|\left(E-T-V_{\pi} \pm i 0^{+}\right)^{-1}\right| \mathbf{r}\right\rangle$ [34,37]. Observing that the bubble diagrams in Fig. 1 are related to $G_{E}^{+}(\mathbf{0}, \mathbf{0})$, while the triangles dressed by Yukawa potentials are related to $\chi_{\mathbf{p}}^{+}(\mathbf{0})$ and $\chi_{\mathbf{p}^{\prime}}^{-}(\mathbf{0})^{*}=\chi_{\mathbf{p}^{\prime}}^{+}(\mathbf{0})$ [34], the LO amplitude reads

$$
\begin{aligned}
\mathcal{A}_{\Delta L=2}^{(\nu)} & =\mathcal{A}_{A}+K_{E^{\prime}} \mathcal{A}_{B}+\overline{\mathcal{A}_{B}} K_{E}+K_{E^{\prime}} \mathcal{A}_{C} K_{E}, \\
K_{E} & =\frac{\chi_{\mathbf{p}}^{+}(\mathbf{0}) \tilde{C}}{1-\tilde{C} G_{E}^{+}(\mathbf{0}, \mathbf{0})},
\end{aligned}
$$

where $\mathcal{A}_{A}, \mathcal{A}_{B}$, and $\mathcal{A}_{C}$ denote the first diagram in the first, second, and third rows of Fig. 1, respectively (without the wave functions at 0 , in the case of $\mathcal{A}_{B}$ and $\mathcal{A}_{C}$ ). $\overline{\mathcal{A}_{B}}$ is similar to $\mathcal{A}_{B}$ and is not shown in Fig. 1.
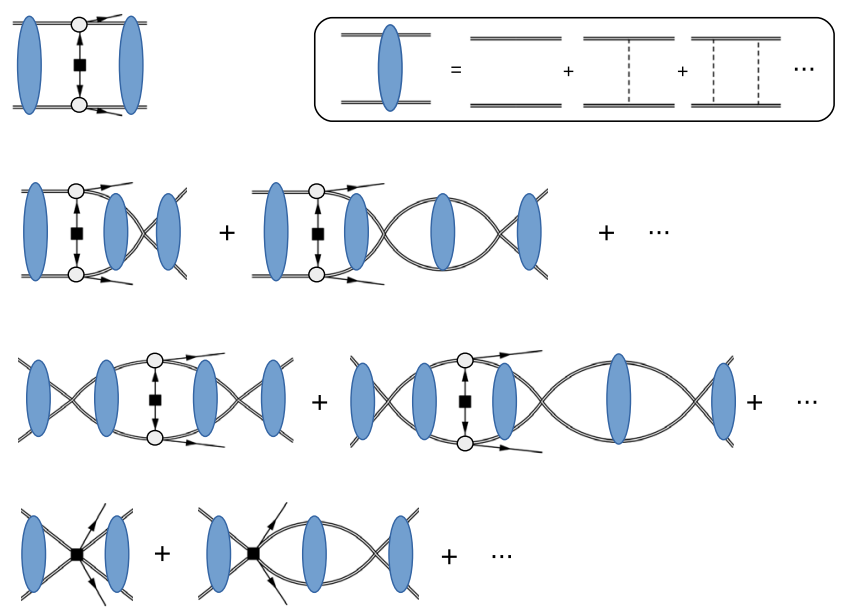

FIG. 1. Diagrammatic representation of LO contributions to $n n \rightarrow$ ppee. Double, dashed, and plain lines denote nucleons, pions, and leptons, respectively. Gray circles denote the nucleon axial and vector currents, and the black square denotes an insertion of $m_{\beta \beta}$. The blue ellipse represents iteration of $V_{\pi}$. In the counterterm amplitude (fourth line) the black square represents $g_{\nu}^{\mathrm{NN}}$. The ellipses in the second to fourth lines denote diagrams with arbitrary numbers of bubble insertions. 
To study the renormalization of the $\Delta L=2$ amplitude, we now discuss the divergence structure of $\mathcal{A}_{\Delta L=2}^{(\nu)} \cdot \chi_{\mathbf{p}}^{+}(\mathbf{0})$ is finite and the divergence in $G_{E}^{+}(\mathbf{0 ,}, \mathbf{0})$ is absorbed by $\tilde{C}^{-1}$, so that $K_{E}$ is finite and scheme independent [34]. We note that (i) all diagrams in $\mathcal{A}_{A}$ are finite. The tree level is finite and each $V_{\pi}$ iteration improves the convergence by bringing in a factor of $d^{3} \mathbf{k} /\left(\mathbf{k}^{2}\right)^{2}$, where one $\mathbf{k}^{2}$ comes from the pion propagator and the other from the two-nucleon propagator. (ii) All the diagrams in $\mathcal{A}_{B}$ and $\overline{\mathcal{A}}_{B}$ are finite. The first loop goes as $d^{3} \mathbf{k} /\left(\mathbf{k}^{2}\right)^{2}$, while $V_{\pi}$ insertions further improve the convergence. (iii) The first two-loop diagram in $\mathcal{A}_{C}$ has a logarithmic divergence, which stems from an insertion of the most singular component of the neutrino potential, namely,

$$
\tilde{V}_{\nu}(\mathbf{q})=\tau^{(1)+} \tau^{(2)+} \frac{1}{\mathbf{q}^{2}}\left(1-\frac{2}{3} g_{A}^{2} \boldsymbol{\sigma}^{(1)} \cdot \boldsymbol{\sigma}^{(2)}\right) .
$$

The two-loop diagram with insertion of $V_{\nu, 0}-\tilde{V}_{\nu}$ and higher-loop diagrams are convergent.

We focus on $\mathcal{A}_{C}$ and write $\mathcal{A}_{C}=\mathcal{A}_{C}^{(\mathrm{div})}+\delta \mathcal{A}_{C}$. In dimensional regularization,

$$
\begin{aligned}
\mathcal{A}_{C}^{(\mathrm{div})} & =-\left(\frac{m_{N}}{4 \pi}\right)^{2}\left(1+2 g_{A}^{2}\right)\left[\Delta+L_{\mathbf{p}, \mathbf{p}^{\prime}}(\mu)\right], \\
L_{\mathbf{p}, \mathbf{p}^{\prime}}(\mu) & =\frac{1}{2}\left(\log \frac{\mu^{2}}{-\left(|\mathbf{p}|+\left|\mathbf{p}^{\prime}\right|\right)^{2}+i 0^{+}}+1\right),
\end{aligned}
$$

where $\Delta \equiv[1 /(4-d)-\gamma+\log 4 \pi] / 2$. The divergence for $d \rightarrow 4$ can be removed by introducing $g_{\nu}^{\mathrm{NN}}$ at LO. The counterterm amplitude, shown in the fourth line of Fig. 1, reads

$$
\mathcal{A}_{\Delta L=2}^{(\mathrm{NN})}=K_{E^{\prime}} \frac{2 g_{\nu}^{\mathrm{NN}}}{\tilde{C}^{2}} K_{E},
$$

and we can renormalize $\mathcal{A}_{\Delta L=2}$ by replacing $\mathcal{A}_{C} \rightarrow \mathcal{A}_{C}+$ $2 g_{\nu}^{\mathrm{NN}} / \tilde{C}^{2}$ in Eq. (6). In the $\overline{\mathrm{MS}}$ scheme,

$\mathcal{A}_{C} \rightarrow \delta \mathcal{A}_{C}+\left(\frac{m_{N}}{4 \pi}\right)^{2}\left[2 \tilde{g}_{\nu}^{\mathrm{NN}}(\mu)-\left(1+2 g_{A}^{2}\right) L_{\mathbf{p}, \mathbf{p}^{\prime}}(\mu)\right]$

after defining the dimensionless coupling

$$
\tilde{g}_{\nu}^{\mathrm{NN}}=\left(\frac{4 \pi}{m_{N} \tilde{C}}\right)^{2} g_{\nu}^{\mathrm{NN}} .
$$

This coupling obeys the renormalization-group equation (RGE)

$$
\mu \frac{d \tilde{g}_{\nu}^{\mathrm{NN}}}{d \mu}=\frac{1}{2}\left(1+2 g_{A}^{2}\right)
$$

confirming that $\tilde{g}_{\nu}^{\mathrm{NN}} \sim \mathcal{O}(1)$. Since $\tilde{C}\left(\mu=m_{\pi}\right) \approx-0.9 / F_{\pi}^{2}$, we find that $g_{\nu}^{\mathrm{NN}} \sim \mathcal{O}\left(F_{\pi}^{-2}\right)$ instead of $\mathcal{O}\left(\left(4 \pi F_{\pi}\right)^{-2}\right)$. A similar enhancement also occurs in four-nucleon couplings induced by higher-dimensional LNV operators. Treating $V_{\pi}$ as a subleading correction [35,39] is equivalent to working to LO in pionless EFT and does not affect our conclusions about the importance of $g_{\nu}^{\mathrm{NN}}$ [26]. Details on how to obtain $\delta \mathcal{A}_{C}$ will be provided in future work [40].

$\mathcal{A}_{\Delta L=2}$ in a cutoff scheme. - The need for a LO counterterm can be demonstrated also in a coordinate-space scheme that makes no direct reference to Feynman diagrams. In this approach, we regulate the short-range part of $V_{0}$ with a smeared $\delta$ function,

$\tilde{C} \delta^{(3)}(\mathbf{r}) \rightarrow \frac{\tilde{C}\left(R_{S}\right)}{\left(\sqrt{\pi} R_{S}\right)^{3}} \exp \left(-\frac{r^{2}}{R_{S}^{2}}\right) \equiv \tilde{C}\left(R_{S}\right) \delta_{R_{S}}^{(3)}(\mathbf{r})$,

and obtain $\psi_{\mathbf{p}^{\prime}}^{-}(\mathbf{r})$ and $\psi_{\mathbf{p}}^{+}(\mathbf{r})$ by solving the Schrödinger equation. We determine $\tilde{C}\left(R_{S}\right)$ by requiring that the ${ }^{1} S_{0}$ scattering length be reproduced $\left(\tilde{C} \approx-0.4 / F_{\pi}^{2}\right.$ at $\left.R_{S}=0.8 \mathrm{fm}\right)$. We find that $1 / \tilde{C}\left(R_{S}\right)$ has linear $\left(1 / R_{S}\right)$ and logarithmic divergences [35] and that the ${ }^{1} S_{0}$ phase shifts at nonzero momentum are indeed $R_{S}$ independent.

We then compute

$$
\mathcal{A}_{\Delta L=2}^{(\nu)}=-\int d^{3} \mathbf{r} \psi_{\mathbf{p}^{\prime}}^{-}(\mathbf{r})^{*} V_{\nu, 0}(\mathbf{r}) \psi_{\mathbf{p}}^{+}(\mathbf{r}),
$$

where $V_{\nu, 0}(\mathbf{r})$ is obtained by Fourier transforming the ${ }^{1} S_{0}$ projection of Eq. (3). In Fig. 2, we plot $\mathcal{A}_{\Delta L=2}^{(\nu)}$ as a function of $R_{S}$. The plot displays a logarithmic dependence on $R_{S}$ [analogous to the $\log \mu$ dependence in Eq. (10)] as well as milder powerlike behavior. Therefore, to obtain a physical, regulator-independent amplitude one needs to include a LO counterterm, given in $r$ space by $V_{\nu, C T}(\mathbf{r})=$ $-2 g_{\nu}^{\mathrm{NN}}\left(R_{S}\right) \delta_{R_{S}}^{(3)}(\mathbf{r})$. The corresponding amplitude,

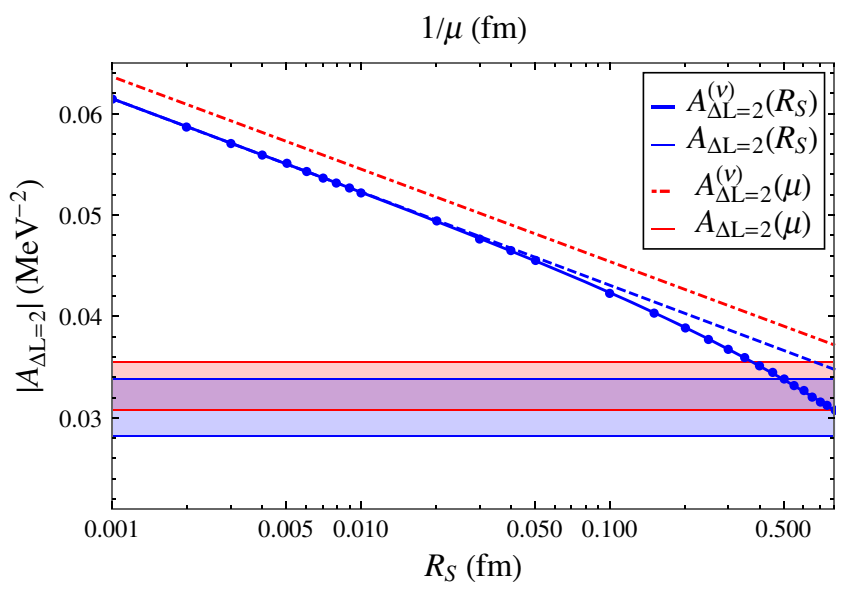

FIG. 2. Matrix element $\mathcal{A}_{\Delta L=2}^{(\nu)}$ for $|\mathbf{p}|=1 \mathrm{MeV}$ and $\left|\mathbf{p}^{\prime}\right|=38 \mathrm{MeV}$, as a function of $R_{S}$. The dashed line shows a fit to $a+b \log R_{S}$, which captures the small $R_{S}$ behavior. The solid line corresponds to a fit that includes $\mathcal{O}\left(R_{S}, R_{S} \log R_{S}\right)$ power corrections. The dash-dotted line shows $\mathcal{A}_{\Delta L=2}^{(\nu)}$ in $\overline{\mathrm{MS}}$ as a function of $1 / \mu$. The horizontal bands represent the total amplitude $\mathcal{A}_{\Delta L=2}$ with $g_{\nu}^{\mathrm{NN}}=\left(C_{1}+C_{2}\right) / 2$, as discussed in the main text. 


$$
\mathcal{A}_{\Delta L=2}^{(\mathrm{NN})}=-\int d^{3} \mathbf{r} \psi_{\mathbf{p}^{\prime}}^{-}(\mathbf{r})^{*} V_{\nu, C T}(\mathbf{r}) \psi_{\mathbf{p}}^{+}(\mathbf{r}),
$$

is also regulator dependent. As expected from Eq. (9), we find its leading divergent behavior to be well reproduced by $1 / \tilde{C}\left(R_{S}\right)^{2}$. We can then make $\mathcal{A}_{\Delta L=2}=\mathcal{A}_{\Delta L=2}^{(\nu)}+\mathcal{A}_{\Delta L=2}^{(\mathrm{NN})}$ finite for $R_{S} \rightarrow 0$ and $R_{S}$ independent by choosing $\tilde{g}_{\nu}^{\mathrm{NN}}\left(R_{S}\right)=-(a / 2)\left(1+2 g_{A}^{2}\right) \log R_{S}+b+c R_{S}+\cdots$, with the coefficient of the logarithm quite close to the $\overline{\mathrm{MS}}$ expectation $a=1$.

Relating $g_{\nu}^{\mathrm{NN}}$ to electromagnetic isospin violation.-The finite part of $g_{\nu}^{\mathrm{NN}}$ can be obtained by matching the chiral EFT amplitude to a lattice QCD calculation performed at the same kinematic point, as it is done in the stronginteracting sector [41]. First lattice results related to doublebeta decay are starting to appear $[42,43]$.

We now discuss a complementary estimate based on the fact that the short-range operators and associated LECs arising in $0 \nu \beta \beta$ and electromagnetic processes are closely related [26]. In the electromagnetic case, the short-range hadronic operators arise from amplitudes in the underlying theory involving two insertions of the electromagnetic current with exchange of hard virtual photons $[44,45]$. In the $\Delta L=2$ case, up to a proportionality factor, the same operators are generated by the insertion of two weak currents with exchange of hard neutrinos. This comes about because the neutrino propagator and weak vertices combine to give a massless gauge-boson propagator in Feynman gauge, multiplied by $8 G_{F}^{2} V_{u d}^{2} m_{\beta \beta} \bar{e}_{L} e_{L}^{c}$ [26]. The LECs needed for $0 \nu \beta \beta$ are therefore related to the LECs associated with the isospin $I=2$ component of the product of two electromagnetic currents, which belongs to the $5_{L} \times$ $1_{R}$ irreducible representation of chiral $\mathrm{SU}(2)_{L} \times \mathrm{SU}(2)_{R}$.

Only two independent four-nucleon operators that transform as $I=2$ objects exist

$$
\begin{aligned}
& O_{1}=\bar{N} \mathcal{Q}_{L} N \bar{N} \mathcal{Q}_{L} N-\frac{\operatorname{Tr}\left[\mathcal{Q}_{L}^{2}\right]}{6} \bar{N} \tau N \cdot \bar{N} \tau N+\{L \leftrightarrow R\}, \\
& O_{2}=2\left(\bar{N} \mathcal{Q}_{L} N \bar{N} Q_{R} N-\frac{\operatorname{Tr}\left[\mathcal{Q}_{L} \mathcal{Q}_{R}\right]}{6} \bar{N} \tau N \cdot \bar{N} \tau N\right),
\end{aligned}
$$

where $\mathcal{Q}_{L}=u^{\dagger} Q_{L} u, \mathcal{Q}_{R}=u Q_{R} u^{\dagger}, u=\exp \left(i \tau \cdot \pi /\left(2 F_{\pi}\right)\right)$, and $Q_{L, R}$ are "spurions" transforming under the chiral group as $Q_{L} \rightarrow L Q_{L} L^{\dagger}, Q_{R} \rightarrow R Q_{R} R^{\dagger}$. In the electromagnetic case, $Q_{L}=Q_{R}=\tau_{3} / 2$, while in $0 \nu \beta \beta Q_{L}=\tau^{+}$, $Q_{R}=0$. In our conventions, $O_{1}$ enters the $\Delta L=2$ Lagrangian with coefficient $2 G_{F}^{2} V_{u d}^{2} m_{\beta \beta} g_{\nu}^{\mathrm{NN}}$. Defining the electromagnetic LECs multiplying $O_{1,2}$ as $e^{2} C_{1,2} / 4$, chiral symmetry dictates $g_{\nu}^{\mathrm{NN}}=C_{1}$.

In the electromagnetic case, $O_{1}$ and $O_{2}$ only differ at the multipion level, and an isospin-breaking two-nucleon observable, such as the $I=2$ combination of scattering lengths $a_{\mathrm{CIB}}=\left(a_{n n}+a_{p p}\right) / 2-a_{n p}$, only constrains the sum $C_{1}+C_{2}$. Extracting this combination from data provides a rough estimate of $g_{\nu}^{\mathrm{NN}}$ under the assumption $C_{1} \sim C_{2}$. As in the $\Delta L=2$ case, we introduce the dimensionless couplings $\tilde{C}_{i} \equiv\left[4 \pi /\left(m_{N} \tilde{C}\right)\right]^{2} C_{i}$ and compute the scattering lengths $a_{p p, n n, n p}$ including the leading sources of isospin breaking - the Coulomb potential and pion mass splitting-and $\tilde{C}_{1}+\tilde{C}_{2}$. Similar to the $\Delta L=2$ case, we find that $\tilde{C}_{1}+\tilde{C}_{2}$ needs to be promoted to LO and obeys the RGE

$$
\mu \frac{d}{d \mu} \frac{\tilde{C}_{1}+\tilde{C}_{2}}{2}=\frac{1}{2}\left(1+g_{A}^{2} \frac{m_{\pi^{+}}^{2}-m_{\pi^{0}}^{2}}{e^{2} F_{\pi}^{2}}\right)
$$

while, of course, $\tilde{C}_{1}$ has the same RGE as $\tilde{g}_{\nu}^{\mathrm{NN}}$. By fitting to $a_{\mathrm{CIB}} \quad$ using $a_{n p}=-23.7 \mathrm{fm}, a_{n n}=-18.9 \mathrm{fm}$, and $a_{p p}=-7.8 \mathrm{fm}$, we find $\left(\tilde{C}_{1}+\tilde{C}_{2}\right) / 2 \approx 2.5$ at $\mu=m_{\pi}$ in the $\overline{\mathrm{MS}}$ scheme. Using instead the $R_{S}$ scheme, we find $\left(\tilde{C}_{1}+\tilde{C}_{2}\right) / 2 \approx 2.0$ at $R_{S}=0.5 \mathrm{fm}$. This estimate, based on data and chiral symmetry, again confirms that $g_{\nu}^{\mathrm{NN}} \sim \mathcal{O}\left(F_{\pi}^{-2}\right)$. Our result is consistent with analyses based on chiral [46-49] and phenomenological potentials such as AV18 [50], which also find that, except at very low energies, long- and short-range components of the CIB interaction induce effects of similar size.

Numerical impact.-To roughly estimate the impact of the contact term, we assume for concreteness $C_{1}=C_{2}$ and hence $g_{\nu}^{\mathrm{NN}}=\left(C_{1}+C_{2}\right) / 2$ at some $\bar{R}_{S}$ or $\bar{\mu}^{-1}$ in the range 0.002-0.8 fm. The total two-nucleon amplitude $\mathcal{A}_{\Delta L=2}=$ $\mathcal{A}_{\Delta L=2}^{(\nu)}+\mathcal{A}_{\Delta L=2}^{(\mathrm{NN})}$ then becomes independent of the regulator, as illustrated in Fig. 2, where the widths of the horizontal bands reflect the ambiguity in the choice of the point $\bar{R}_{S}$ or $\bar{\mu}$ where $C_{1}=C_{2}$ is assumed. (They do not account for the uncontrolled error of the assumption itself.) The relative size of the two components depends on $R_{S}$, with $\mathcal{A}_{\Delta L=2}^{(\mathrm{NN})} / \mathcal{A}_{\Delta L=2}^{(\nu)} \sim 30 \%$ at $R_{S} \sim 0.1 \mathrm{fm}$, decreasing to $\sim 10 \%$ at $R_{S} \sim 0.6 \mathrm{fm}$. More insight can be obtained from the matrix-element densities $\rho_{\nu}$ and $\rho_{\mathrm{NN}}$, implicitly defined from Eqs. (14) and (15) by

$\mathcal{A}_{\Delta L=2}^{(\nu)}=\int d r \rho_{\nu}(r), \quad \mathcal{A}_{\Delta L=2}^{(\mathrm{NN})}=\int d r \rho_{\mathrm{NN}}(r)$.

Figure 3 (top panel) shows that $\rho_{\mathrm{NN}}(r)$ is concentrated at smaller distances than $\rho_{\nu}(r)$, and its contribution to the amplitude is thus partially diluted.

We have performed a similar analysis for $A=6,12$ nuclei, using variational Monte Carlo nuclear wave functions [51] based on the AV18 two-nucleon [50] and IL7 three-nucleon [52] interactions. The mismatch between the short-range behaviors of existing strong-interaction potentials and our $0 \nu \beta \beta$ interaction introduces additional model dependence, which we mitigate by (i) considering an alternative extraction of $\left(C_{1}+C_{2}\right) / 2$ from the phase-shift analysis of Refs. [47,48], which employs the same 


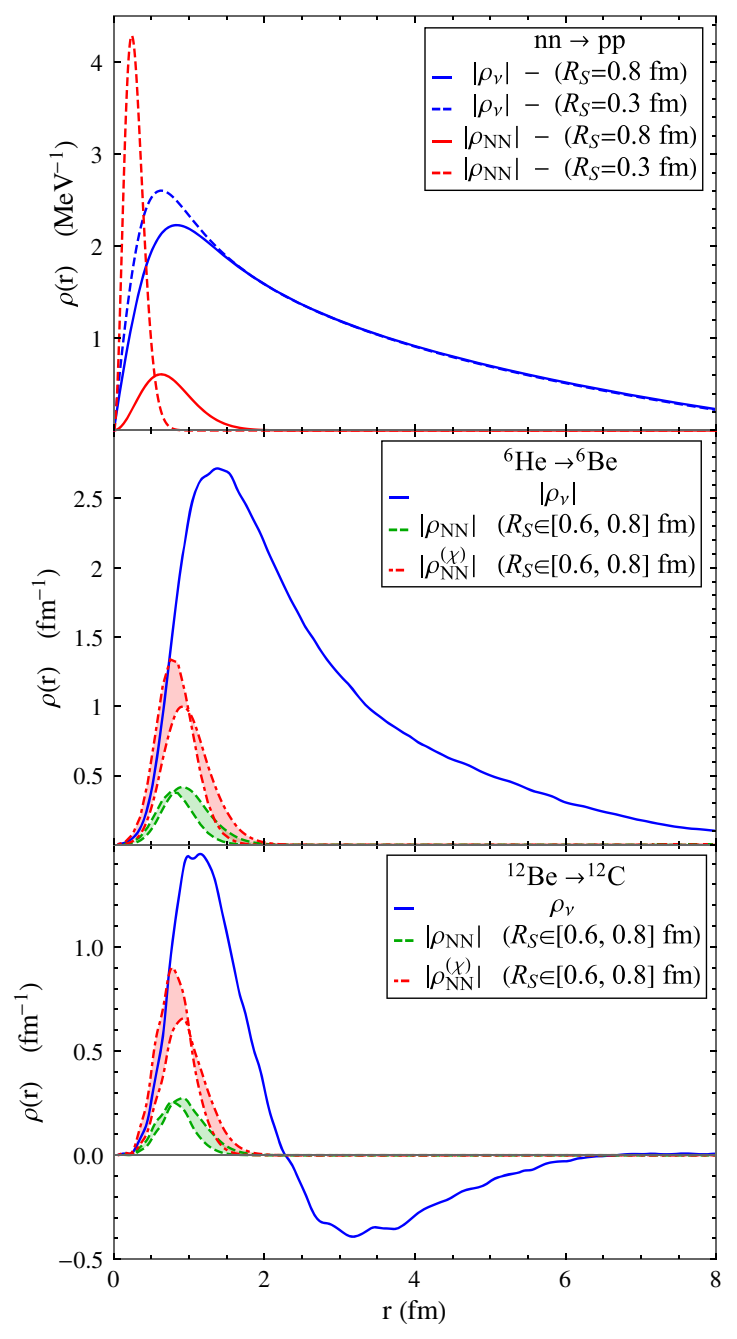

FIG. 3. $\rho_{\nu}(r)$ and $\rho_{\mathrm{NN}}(r)$ for the $n n \rightarrow$ ppee process (top) and for nuclear transitions with $A=6$ (middle) and $A=12$ (bottom). In the middle and bottom panels, the green $\left(\rho_{\mathrm{NN}}\right)$ and red $\left(\rho_{\mathrm{NN}}^{(\chi)}\right)$ bands correspond to $g_{\nu}^{\mathrm{NN}}=\left(C_{1}+C_{2}\right) / 2$ extracted from our analysis and from Refs. [47,48], respectively.

regulator (13) with $R_{S} \simeq 0.6-0.8 \mathrm{fm}$, approximately the range of AV18's short-range part, and (ii) simply replacing our $V_{\nu, C T}(\mathbf{r})$ with AV18's short-range CIB potential. Note that $C_{1}+C_{2}$ is related to the CIB coefficient $C_{0}^{I T}$ of Refs. $[47,48]$ by $\left(C_{1}+C_{2}\right) / 2=-6 C_{0}^{I T} / e^{2}$.

For $\Delta I=0$ transitions such as the ${ }^{6} \mathrm{He} \rightarrow{ }^{6} \mathrm{Be}$ shown in Fig. 3 (middle panel), we find $\mathcal{A}_{\Delta L=2}^{(\mathrm{NN})} / \mathcal{A}_{\Delta L=2}^{(\nu)} \sim 10 \%$, similar to the $n n \rightarrow$ ppee case. In realistic $0 \nu \beta \beta$ transitions, however, the total nuclear isospin changes by two units, $\Delta I=2$. This implies the presence of a node in $\rho_{\nu}(r)$ due to the orthogonality of the initial and final spatial wave functions. The resulting partial cancellation between the regions with $r \lesssim 2 \mathrm{fm}$ and $r \gtrsim 2 \mathrm{fm}$ [51] leads to a relative enhancement of the short-range contribution, as illustrated in Fig. 3 (bottom panel) for ${ }^{12} \mathrm{Be} \rightarrow{ }^{12} \mathrm{C}$. Numerically, we find $\mathcal{A}_{\Delta L=2}^{(\mathrm{NN})} / \mathcal{A}_{\Delta L=2}^{(\nu)} \sim 25 \%$ (our fit), $\sim 55 \%$ (fit from Refs. [47,48]), and $260 \%$ (AV18 representation of the short-range CIB potential). Because (i) the node in the density is a robust feature of $\Delta I=2$ transition $[53,54]$, and (ii) the relative size of the matrix elements of short- and long-range operators is roughly the same in nuclei from $A=10$ through $A=136$ [51], we expect the effects in ${ }^{12} \mathrm{Be} \rightarrow{ }^{12} \mathrm{C}$ and experimentally relevant transitions to be of comparable size.

Conclusion.-The above arguments suggest that the new leading-order short-range $\Delta L=2$ potential identified in this Letter can affect the $0 \nu \beta \beta$ amplitude and, consequently, the quantitative implications of experiments on $m_{\beta \beta}$ at the $\mathcal{O}(1)$ level. (At subleading orders, a similar analysis of the renormalization of the $0 \nu \beta \beta$ amplitude must be performed, which takes into account the required modifications to Weinberg's power counting in the strong-interaction sector, such as the perturbative correction induced by the ${ }^{1} S_{0}$ effective range at next-to-leading order [37].) In particular, sensitivity to the inverted hierarchy [24] in next-generation experiments might be increased or much decreased, depending on the size and sign of the interference between the new short-range contribution and the long-range term. We hope this will stimulate work towards a more controlled determination of $g_{\nu}^{\mathrm{NN}}$ from lattice QCD and an assessment of the impact of the short-range potential in nuclei of experimental interest.

V. C., W. D., M. G., E. M., and S. P. acknowledge support by the U.S. Department of Energy, Office of Science, and by the LDRD program at Los Alamos National Laboratory. V. C. and E. M. acknowledge partial support from the DOE topical collaboration on "Nuclear Theory for Double-Beta Decay and Fundamental Symmetries." J. d. V. acknowledges support by the Dutch Organization for Scientific Research (NWO) through a VENI grant. The work of U.v.K. was supported in part by the U.S. Department of Energy, Office of Science, Office of Nuclear Physics, under Award No. DE-FG0204ER41338, and by the European Union Research and Innovation program Horizon 2020 under Grant No. 654002. We acknowledge stimulating discussions with Will Detmold and Martin Savage, which triggered this research. We thank Joe Carlson, Jon Engel, Amy Nicholson, Maria Piarulli, Petr Vogel, Andre WalkerLoud, and Bob Wiringa for discussions at various stages of this work.

[1] J. Schechter and J. W. F. Valle, Phys. Rev. D 25, 2951 (1982).

[2] P. Minkowski, Phys. Lett. 67B, 421 (1977).

[3] R. N. Mohapatra and G. Senjanovic, Phys. Rev. Lett. 44, 912 (1980).

[4] M. Gell-Mann, P. Ramond, and R. Slansky, in Supergravity Workshop Stony Brook, New York, September 27-28, 1979, Conference Proceedings, Vol. C790927 (1979), p. 315. 
[5] S. Davidson, E. Nardi, and Y. Nir, Phys. Rep. 466, 105 (2008).

[6] A. Gando et al. (KamLAND-Zen Collaboration), Phys. Rev. Lett. 110, 062502 (2013).

[7] M. Agostini et al. (GERDA Collaboration), Phys. Rev. Lett. 111, 122503 (2013).

[8] J. B. Albert et al. (EXO-200 Collaboration), Nature (London) 510, 229 (2014).

[9] S. Andringa et al. (SNO+ Collaboration), Adv. High Energy Phys. 2016, 1 (2016).

[10] A. Gando et al. (KamLAND-Zen Collaboration), Phys. Rev. Lett. 117, 082503 (2016); 117, 109903(E) (2016).

[11] S. R. Elliott et al., arXiv:1610.01210.

[12] M. Agostini et al., Nature (London) 544, 47 (2017).

[13] J. B. Albert et al. (EXO Collaboration), Phys. Rev. Lett. 120, 072701 (2018).

[14] C. Alduino et al. (CUORE Collaboration), Phys. Rev. Lett. 120, 132501 (2018).

[15] O. Azzolini et al. (CUPID-0 Collaboration), arXiv: 1802.07791.

[16] S. Weinberg, Phys. Rev. Lett. 43, 1566 (1979).

[17] K. S. Babu and C. N. Leung, Nucl. Phys. B619, 667 (2001).

[18] G. Prezeau, M. Ramsey-Musolf, and P. Vogel, Phys. Rev. D 68, 034016 (2003).

[19] A. de Gouvêa and J. Jenkins, Phys. Rev. D 77, 013008 (2008).

[20] L. Lehman, Phys. Rev. D 90, 125023 (2014).

[21] M. L. Graesser, J. High Energy Phys. 08 (2017) 099.

[22] V. Cirigliano, W. Dekens, J. de Vries, M. L. Graesser, and E. Mereghetti, J. High Energy Phys. 12 (2017) 082.

[23] S. M. Bilenky and S. T. Petcov, Rev. Mod. Phys. 59, 671 (1987); 60, 575(E) (1988).

[24] S. M. Bilenky and C. Giunti, Int. J. Mod. Phys. A 30, 1530001 (2015).

[25] J. Engel and J. Menéndez, Rep. Prog. Phys. 80, 046301 (2017).

[26] V. Cirigliano, W. Dekens, E. Mereghetti, and A. Walker-Loud, arXiv:1710.01729.

[27] P. F. Bedaque and U. van Kolck, Annu. Rev. Nucl. Part. Sci. 52, 339 (2002).

[28] E. Epelbaum, H.-W. Hammer, and U.-G. Meißner, Rev. Mod. Phys. 81, 1773 (2009).

[29] R. Machleidt and D. R. Entem, Phys. Rep. 503, 1 (2011).

[30] S. Weinberg, Phys. Lett. B 251, 288 (1990).

[31] S. Weinberg, Nucl. Phys. B363, 3 (1991).

[32] N. Cabibbo, Phys. Rev. Lett. 10, 531 (1963).
[33] M. Kobayashi and T. Maskawa, Prog. Theor. Phys. 49, 652 (1973).

[34] D. B. Kaplan, M. J. Savage, and M. B. Wise, Nucl. Phys. B478, 629 (1996).

[35] S. R. Beane, P. F. Bedaque, M. J. Savage, and U. van Kolck, Nucl. Phys. A700, 377 (2002).

[36] A. Nogga, R. G. E. Timmermans, and U. van Kolck, Phys. Rev. C 72, 054006 (2005).

[37] B. Long and C.-J. Yang, Phys. Rev. C 86, 024001 (2012).

[38] M. Pavón Valderrama and D. R. Phillips, Phys. Rev. Lett. 114, 082502 (2015).

[39] D. B. Kaplan, M. J. Savage, and M. B. Wise, Nucl. Phys. B534, 329 (1998).

[40] V. Cirigliano, W. Dekens, J. de Vries, M. L. Graesser, E. Mereghetti, S. Pastore, and U. van Kolck (to be published).

[41] N. Barnea, L. Contessi, D. Gazit, F. Pederiva, and U. van Kolck, Phys. Rev. Lett. 114, 052501 (2015).

[42] A. Nicholson, E. Berkowitz, C. C. Chang, M. A. Clark, B. Joo, T. Kurth, E. Rinaldi, B. Tiburzi, P. Vranas, and A. Walker-Loud, Proc. Sci., LATTICE2016 (2016) 017.

[43] P. E. Shanahan, B. C. Tiburzi, M. L. Wagman, F. Winter, E. Chang, Z. Davoudi, W. Detmold, K. Orginos, and M. J. Savage, Phys. Rev. Lett. 119, 062003 (2017).

[44] B. Moussallam, Nucl. Phys. B504, 381 (1997).

[45] B. Ananthanarayan and B. Moussallam, J. High Energy Phys. 06 (2004) 047.

[46] M. Walzl, U.-G. Meißner, and E. Epelbaum, Nucl. Phys. A693, 663 (2001).

[47] M. Piarulli, L. Girlanda, R. Schiavilla, R. Navarro Pérez, J. E. Amaro, and E. Ruiz Arriola, Phys. Rev. C 91, 024003 (2015).

[48] M. Piarulli, L. Girlanda, R. Schiavilla, A. Kievsky, A. Lovato, L. E. Marcucci, S. C. Pieper, M. Viviani, and R. B. Wiringa, Phys. Rev. C 94, 054007 (2016).

[49] P. Reinert, H. Krebs, and E. Epelbaum, arXiv:1711.08821.

[50] R. B. Wiringa, V. G. J. Stoks, and R. Schiavilla, Phys. Rev. C 51, 38 (1995).

[51] S. Pastore, J. Carlson, V. Cirigliano, W. Dekens, E. Mereghetti, and R. B. Wiringa, Phys. Rev. C 97, 014606 (2018).

[52] S. C. Pieper, AIP Conf. Proc. 1011, 143 (2008).

[53] F. Šimkovic, A. Faessler, V. Rodin, P. Vogel, and J. Engel, Phys. Rev. C 77, 045503 (2008).

[54] J. Menéndez, A. Poves, E. Caurier, and F. Nowacki, Nucl. Phys. A818, 139 (2009). 